\title{
A SPACE BORNE LIDAR INSTRUMENT FOR METHANE: INSTRUMENT MONITORING \& QUALITY ASSURANCE
}

\author{
Mourad Hamidouche, G. Lichtenberg, B. Aberle, and T. Trautmann \\ Remote Sensing Technology Institute, German Aerospace Center (DLR), 82234 Wessling, Germany
}

\begin{abstract}
The concentration of methane $\left(\mathrm{CH}_{4}\right)$ with other greenhouse gases in the atmosphere lead to the raise of the mean Earth surface temperature. Methane is in fact the most important greenhouse gas in the atmosphere after $\mathrm{H}_{2} \mathrm{O}$ and $\mathrm{CO}_{2}$, with emission from anthropogenic and natural processes. Global observations and monitoring with high accuracy and spatial resolution to track down its sources and sinks on a global scale are however necessary, but yet challenging.
\end{abstract}

The active space borne Lidar instrument MERLIN (Methane Remote Sensing Lidar Mission) will provide new sensitive measurements of methane distribution with unprecedented quality, with a precision $<2 \%$. In this paper, we present our approach to perform a key ground segment task that will support MERLIN scientific activities which is the long-term monitoring of the Lidar instrument and its performance. The instrument long term monitoring task will track the measurements quality and the performance of the instrument and its subsystems on a daily up to a monthly basis over MERLIN mission lifetime.

Key words: Methane $\left(\mathrm{CH}_{4}\right)$; MERLIN; Lidar; Atmosphere; Satellite.

\section{INTRODUCTION}

Methane is the most important greenhouse gas in the atmosphere after $\mathrm{H}_{2} \mathrm{O}$ and $\mathrm{CO}_{2}$, with emission from anthropogenic and natural processes. Several airborne and space borne instruments (e.g. GOSAT, TROPOMI) provided important results based on their atmospheric methane measurements [1].

The new generation space borne Lidar mission MERLIN (Methane Remote Sensing Lidar Mission) will make very sensitive measurements of atmospheric methane $\left(\mathrm{CH}_{4}\right)$ distribution with unprecedented quality and coverage. It will provide $50 \mathrm{~km}$ averaged methane column concentrations. It will therefore offer the necessary sensitivity to probe methane fluxes, over different key geographical areas, with a precision better than 2\% [2]. MERLIN will eventually track down the methane sources and sinks on a global scale.

The instrument will be mounted on a satellite platform flying at $\sim 500 \mathrm{~km}$ altitude in a Sun synchronous orbit [3]. The instrument will continuously send and receive a pair of pulses at two different wavelengths. One wavelength $\lambda_{\text {on }}=$ $1645.552 \mathrm{~nm}$ is centered on $\mathrm{CH}_{4}$ absorption line. The other pulse is a reference at $\lambda_{o f f}=1645.846 \mathrm{~nm}$ which is slightly shifted by a few tenth of $\mathrm{nm}$. It is located where $\mathrm{CH}_{4}$ absorption is relatively smaller but yet it is close enough to have a nearly identical interaction with the atmosphere and the reflecting surface. The laser footprint size (diameter) is about 150 meters [3]. From the back-scattered signals of 
both pulses (on and off) the DAOD (Differential Absorption Optical Depth) of methane is measured, and thus its density.

\section{INSTRUMENT MONITORING}

\subsection{Motivation}

The instrument the long term monitoring (LTM) is an offline function that will track the behavior of the instrument and its subsystems. It will also monitor the instrument's performance in response to expected or unexpected natural events or technical situations. This is achieved by analyzing the measurement data (L0, L1, L2) and housekeeping information over different time frames. The corresponding time scales may vary for different functions and parameters (e.g. weekly or monthly depending on the parameter). The timescales can also change when deemed necessary by the LTM team. If a specific parameter shows unexpected temporal deviation, it would likely require further monitoring with shorter time scales; and therefore allowing the user to probe and constrain the origin of the detected deviation. With LTM function, we would ensure the health of the instrument in the long run (e.g. detection of long-term drifts) and may as well anticipate any deterioration effects. Even though instrument health and safety are not considered one of the main goals of a long term monitoring function, instrument health degradation might appear gradually and therefore could only be noticed over long time frames and not in real time.

Ultimately, long term monitoring will generate new feedback to assist existing and/or future data/scientific analysis and the calibration processes. The results of LTM could imply some modifications, such as:

- Modification of payload parameters

- Modification of calibration data processing tools

- Modification of operation schedule

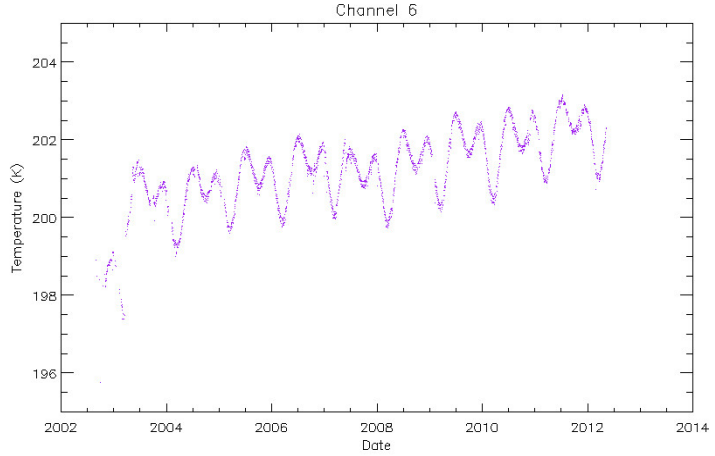

Figure 1: Temperature variation of SCIAMACHY instrument detector in the shortwave-infrared channel 6 as a function of time in years. It varied periodically or seasonally by a few tenths of a Kelvin, while on average it slightly increased over the years by 2 to 3 Kelvin.

\subsection{Key monitored MERLIN components:}

- Detector Response: The detector temperature, power supply, and dark current will be continuously monitored to identify any trends (either peculiar or not). Figure 1 shows an example of a long term monitoring result of SCIAMACHY. It shows the detector temperature in the shortwave-infrared channel 6 [4] where a variation of $0.29 \mathrm{~K} /$ year can be perceived. In MERLIN, similar trends might occur in any of the instruments subsystems. Additionally a degradation over time can also occur, and that could have various origins, such as cosmic radiation or hard UV. The long term monitoring time-horizons are long enough to detect and thus probe any degradation trend or any periodical peculiarity.

- The Laser: The emitted $\left(\mathrm{T}_{x}\right)$ and the backscattered or returned $\left(\mathrm{R}_{x}\right)$ laser pulses will be monitored including their features and values (e.g. travel time, shape, energy, center bin position) in order to avoid inaccuracies or misinterpretation of methane measurements. $\mathrm{R}_{x}$ is in fact expected to have different shapes per shot depending on the line of sight. We will therefore make analytical models to check the consistency of the measured properties of each pulse 


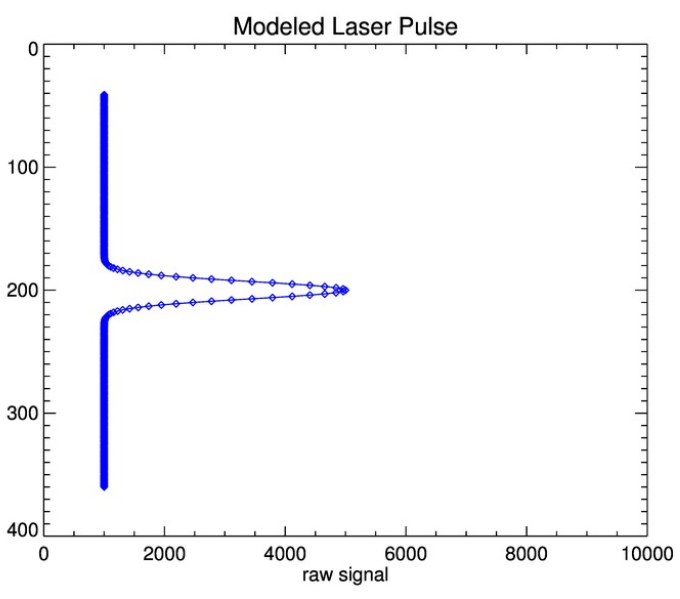

Figure 2: A modeled laser pulse without distortions. The $\mathrm{y}$-axis represents the temporal bins number. The pulse peak value is $\sim 5000$ and the noisy baseline is $\sim 1000$ arbitrary unit. The corresponding SNR (signal to noise ratio) will be permanently tracked as a function of the traveled projected distance.

(Figure 2). Furthermore, the measured backscattered $\left(\mathrm{R}_{x}\right)$ energies in level 0 data over certain regions on Earth (e.g. sea), that have relatively stable reflectivity over time, should be close and correlated with the energies from level 1. A nearly linear correlation between the two (level $0 \&$ level 1) energy measurements would be expected [5]. Whether the linear correlation is achieved or not, these correlation tests will provide a unique opportunity to analyze and monitor various components from level 0 to level 1.

- Geolocation: Tracking the Telescope/Instrument tilts in two coordinates is key to validate various components, as any minor (roll) tilt would affect the on ground Laser footprint shape and location and thus the geolocation. Any small (pitch) motion might add Doppler shifts that would affect $\mathrm{CH}_{4}$ column density measurements. Monitoring the laser footprint location could be achieved by routinely targeting known ground targets [5]. The signal intensity should drop and rise when the footprint crosses or transits over pre-defined ground regions such as land/water (e.g.

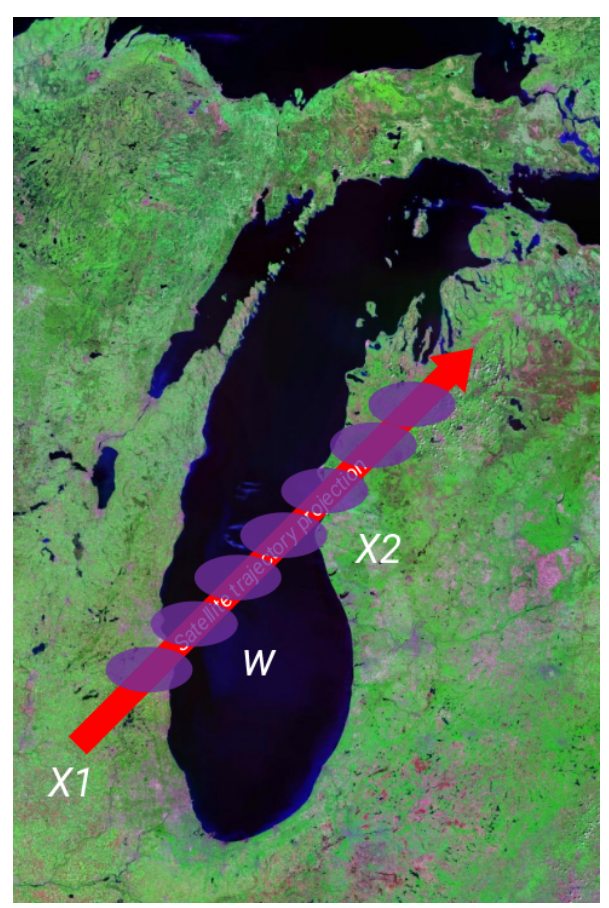

Figure 3: Footprint [purple circles drawn not to scale] position calculation using a lake (example of Lake Michigan, USA). The red arrow represents the satellite projected trajectory. The position determination is achieved by comparing the signal difference at the transition region land/water $(\mathrm{X} 1 / \mathrm{w})$ or the opposite water/land (w/X2).

Figure 3). We would therefore compare our signals during the crossing event to check the actual footprint position compared to the expected one. For the same purpose, we would additionally, and where available [5], compare our MERLIN backscattered values against the measured reflectance from MODIS (Moderate Resolution Imaging Spectroradiometer) instrument [6] in its band 6 at methane wavelength. Any footprint position deviation or jittering would be analyzed for the measurements' validation and the geolocation as well as for the instrument components alignment monitoring. 


\section{SUMMARY}

Long term monitoring will be a key ground segment component supporting MERLIN scientific activities. It will mainly contribute into the data verification and validation over the mission lifetime (e.g. Instrument pointing, Laser performance). LTM will add specific insights on the instrument behavior and its data in order to avoid scientific measurement biases, by tracking any effects due to unpredicted instrument behavior or unexpected natural events (e.g. solar flares). On the other hand, it will investigate the stability of the laser and also routinely evaluate the geolocation to ensure the reliability of the measurements of $\mathrm{CH}_{4}$ density, and thus the overall scientific results.

\section{REFERENCES}

[1] Hu, H.; Landgraf, J.; Detmers, R.; Borsdorff, T.; Aan de Brugh, J.; Aben, I.; Butz, A.; Hasekamp, O.; 2018; Geophysical Research Letters, Volume 45, Issue 8, pp. 3682-3689

[2] Kiemle, C.; Kawa, S. R.; Quatrevalet, M.; Browell, E. V.; 2014; Journal of Geophysical Research: Atmospheres, Volume 119, Issue 7, pp. 4365-4379

[3] Ehret, G. et al.; 2017; Remote Sensing, Volume 9, Issue 10, p. 1052

[4] Gottwald, M.; Krieg, E.; Lichtenberg, G.; Noël, S.; Bramstedt, K.; Bovensmann, H.; Snel, R.; Krijger, M. SCIAMACHY In-Orbit Operations and Performance; SCIAMACHY Mission Documents; Springer: Berlin, Germany, 2016; PO-TN-DLR-SH-0034

[5] Hamidouche, M.; Lichtenberg, G.; Aberle, B.; Trautmann, T.; 2017; EUMETSAT Meteorological Satellite Conference Proceedings; Atmospheric composition: recent advances in satellite products and applications

[6] Vermote, E. F.; Kotchenova, S.; 2008; Journal of Geophysical Research: Atmospheres, Volume 113, Number D12, p. D23S90 